
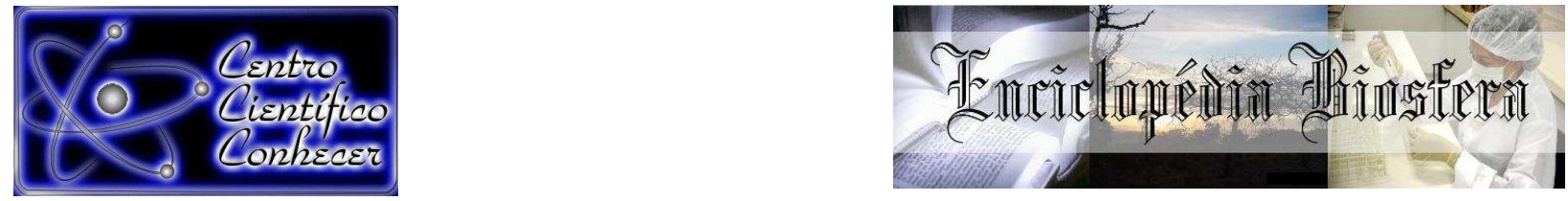

\title{
AVALIAÇÃO DA QUALIDADE FISIOLÓGICA E SANITÁRIA DE SEMENTES DE SOJA TRATADAS COM EXTRATO VEGETAL
}

Niédja Marizze Cezar Alves ${ }^{1}$, Rayane Letícia de Oliveira Castro ${ }^{2}$, Nahyara Batista Caires Galle ${ }^{2}$, Maria Isabel Postil da Silva ${ }^{2}$

1. Professora Doutora da Universidade Federal de Mato Grosso (niedjamarizze@yahoo.com.br) Rondonópolis-Brasil

2. Graduanda do curso de Engenharia Agrícola e Ambiental da Universidade Federal de Mato Grosso, Rondonópolis-Brasil

Recebido em: 08/09/2015 - Aprovado em: 14/11/2015 - Publicado em: 01/12/2015

DOI: http://dx.doi.org/10.18677/Enciclopedia_Biosfera_2015_259

\begin{abstract}
RESUMO
Objetivou-se com esse trabalho avaliar a qualidade fisiológica e sanitária de sementes de soja tratadas com extrato vegetal de pimenta-do-reino armazenadas em embalagem do tipo politereftalato de etileno (PET) por distintos períodos de armazenamento. Para o experimento foram utilizados, no tratamento das sementes, doses de 0 (controle), 10 e $20 \mathrm{~mL}$ do extrato hidroalcoólico de pimenta-do-reino (extrato:água), para cada $300 \mathrm{~g}$ de sementes. As sementes foram avaliadas mensalmente quanto à umidade (\%), micoflora (\%) e germinação (\%). Utilizou-se o delineamento inteiramente casualizado (DIC) disposto em esquema fatorial (3 doses em $\mathrm{mL}$ do extrato x 5 tempos de armazenamento) com quatro repetições. Foram identificados os fungos Penicillium sp. e Aspergillus flavus; o extrato apresentou controle eficaz na dose de $10 \mathrm{~mL}$ do extrato até 60 dias de armazenamento. Os resultados permitem afirmar que uso do extrato de pimenta do reino é benéfico para a utilização na agricultura apresentando um baixo custo de produção e fácil manejo.
\end{abstract}

PALAVRAS-CHAVE: Piper nigrum, qualidade fisiológica, controle alternativo, Glycine max L.

\section{EVALUATION OF PHYSIOLOGICAL AND SANITARY QUALITY OF SOYBEAN SEEDS TREATED WITH PLANT EXTRACT}

\begin{abstract}
The aim of this work was to evaluate the physiological and sanitary quality of soybean seeds treated with plant extract of black pepper stored in packing type polyethylene terephthalate (PET) for different periods of storage. For the experiment were used to treat seeds doses of 0 (control), 10 and $20 \mathrm{~mL}$ of hydroalcoholic extract of black pepper (extract:water) for every $300 \mathrm{~g}$ of seeds. The seeds were evaluated monthly as to their humidity (\%), mycoflora (\%) and germination (\%). Completely randomized design (CRD) was used arranged in a factorial design (3 doses in $\mathrm{mL}$ of extract $\times 5$ periods of storage) with four replications. Fungi Penicillium sp. and Aspergillus flavus were identified; the extract effectively controlled dose of $10 \mathrm{~mL}$ of
\end{abstract}


the extract until 60 days of storage. The results indicate that use of black pepper extract is beneficial for use in agriculture presenting a low production cost and easy handling.

KEYWORDS: Piper nigrum, physiological quality, alternative control, Glycine max L.

\section{INTRODUÇÃO}

A soja (Glycine max (L.) Merril) destaca-se por apresentar um alto valor proteico, além de um baixo teor de gordura saturada, ausência de colesterol e uma quantidade considerável de fibras e minerais, complementando a dieta humana e animal (SILVA et al., 2006). Trata-se de uma leguminosa de grande expressão no cenário mundial (CONAB, 2015).

É uma cultura que apresenta, a exemplo de outras, inúmeros problemas quanto à obtenção de sementes de alta qualidade. A contaminação por fungos pode ocorrer na formação das sementes e nas tradicionais práticas de colheita, secagem, beneficiamento e armazenamento. Diante dessa situação, os tratamentos sanitários muitas vezes são indispensáveis, pois visam à eliminação desses patógenos que possam comprometer sua germinação e vigor (SCHNEIDER et al., 2015).

A etapa de armazenamento tem como objetivo básico manter o nível de qualidade fisiológica das sementes até sua utilização na semeadura (CARVALHO; NAKAGAWA, 2012). As informações a respeito do comportamento das sementes diante das prováveis condições climáticas que ocorrem durante a conservação, podem auxiliar na tomada de decisão sobre o armazenamento do produto com base na relação custo-benefício, decorrente de possíveis perdas de qualidade na estocagem (SMANIOTTO et al., 2014).

Durante o armazenamento, os principais fatores que influenciam a viabilidade de sementes de soja são temperatura, umidade relativa do ar e o teor de água na semente (SMANIOTTO et al., 2014). O controle dos microrganismos durante o armazenamento, em especial os fungos, é feito preferencialmente com fungicidas, sendo muitos deles prejudiciais ao homem e ao meio ambiente. Entretanto, a agricultura atual tem buscado formas alternativas para o tratamento de sementes e controle de doenças, que visem minimizar impactos ambientais (DEUNER et al., 2015). Neste contexto, tem se destacado as pesquisas com alternativas de controle natural relacionadas à preservação da fauna e da flora (MIGLIORINI et al., 2012), sendo o extrato de pimenta-do-reino um produto potencial para tal finalidade.

Diante do exposto, objetivou-se avaliar a qualidade fisiológica e sanitária das sementes de soja tratadas com extrato vegetal de pimenta-do-reino e armazenadas em embalagem do tipo PET.

\section{MATERIAL E MÉTODOS}

As sementes de soja (Glycine max L.) apresentaram teor de água de 8,90\% (b.u.), germinação de $100 \%$ e $0,0 \%$ de infecção por fungos. Antecedendo o armazenamento das sementes em embalagens do tipo PET, um lote com aproximadamente $5 \mathrm{~kg}$ foi tratado com extrato alcoólico de pimenta-do-reino. Este extrato foi obtido pesando-se inicialmente $500 \mathrm{~g}$ de massa triturada de pimenta-doreino (pó obtido do grão), que foi umedecido em um béquer com líquido extrator (500 $\mathrm{mL}$ de álcool $70 \%$ ). Esta massa umedecida foi aos poucos transferida para o interior de um recipiente com tampa, onde permaneceu por um período de $48 \mathrm{~h}$. Passado o período de maceração, a massa umedecida foi coada em papel de filtro e em 
seguida guardada em recipiente âmbar. A aplicação do extrato foi realizada manualmente.

Foram utilizados, no tratamento das sementes, doses de 0 (controle), 10 e 20 $\mathrm{mL}$ (extrato:água) do extrato hidroalcoólico de pimenta-do-reino, para cada $300 \mathrm{~g}$ de sementes. Em seguida, as sementes foram secas a temperatura ambiente por um período de $24 \mathrm{~h}$, sobre folhas de papel jornal. As sementes tratadas foram acondicionadas em recipientes de PET onde permaneceram armazenadas por 5 meses.

As sementes foram avaliadas mensalmente quanto a teor de umidade (\%), micoflora (\%) e germinação (\%).

Para determinação da umidade utilizou-se o método padrão de estufa a $105 \pm$ $2{ }^{\circ} \mathrm{C}$, com quatro subamostras de $20 \mathrm{~g}$ acondicionadas em placas de petri, previamente secados, pesados e colocados em estufa pelo tempo de $24 \mathrm{~h}$ (BRASIL, 2009). Após este período os recipientes foram retirados da estufa e postos em um dessecador, durante $45 \mathrm{~min}$. Em seguida, as sementes foram novamente pesadas obtendo-se a porcentagem de peso expresso em base úmida.

O método de incubação utilizado foi o blotter-test ou o método do papel de filtro (NEERGAARD, 1979). As sementes, em número de dez, foram colocadas no interior de placas de Petri sobre substrato constituído de dois discos sobrepostos de papel germitest umedecidos com água esterilizada. Posteriormente, as placas de Petri contendo as sementes foram deixadas em uma estufa incubadora, tipo B.O.D. a $25 \pm 1 \stackrel{\circ}{ } 2$. No sétimo dia de incubação, as sementes foram examinadas individualmente, para visualização de colônias, identificação e contagem de fungos. A quantificação da micoflora foi feita considerando-se as porcentagens por amostras avaliadas das sementes contendo fungos.

Realizou-se o teste de germinação em laboratório utilizando-se quatro repetições de 50 sementes semeadas em folhas de papel germitest, umedecidas em água destilada, na proporção de três vezes a massa do papel seco. Rolos foram confeccionados e acomodados em recipientes plástico para evitar perda de umidade, e posteriormente, colocados no germinador (BOD) regulado a temperatura de $25 \pm 1 \stackrel{\circ}{ } \mathrm{C}$ (BRASIL, 2009). Os critérios adotados, na classificação das plântulas normais e anormais, foram as descritas pelas normas do Ministério da Agricultura (BRASIL, 2009).

Utilizando-se o delineamento inteiramente casualizado (DIC) disposto em esquema fatorial. A significância do efeito dos tratamentos foi determinada por meio do Teste $F$, sendo as médias do fator concentração do extrato hidroalcoólico comparadas pelo Teste de Tukey $(p<0,05)$. Para o fator períodos de armazenamento ajustou-se regressões polinomiais.

\section{RESULTADOS E DISCUSSÃO}

De acordo com os resultados encontrados (Tabela 1), observou-se que 0 extrato aquoso de sementes de pimenta-do-reino interferiu na porcentagem de germinação das sementes de soja. A diminuição iniciou-se com a dose de $20 \mathrm{~mL}$ do extrato no tempo de 60 dias. Essa redução foi de 7,5 pontos percentuais frente à 0 $\mathrm{mL}$ (testemunha) e de 11 pontos percentuais em relação à dose de $10 \mathrm{~mL}$, a qual manteve, para este tempo, superioridade.

Para o tempo de 90 dias, observa-se que o extrato de pimenta-do-reino não foi eficiente na manutenção da viabilidade das sementes de soja armazenadas, ou seja, tanto a dose de $10 \mathrm{~mL}$ quanto a dose de $20 \mathrm{~mL}$ apresentaram resultados inferiores em relação à testemunha. Essa perda na germinação foi mais acentuada, 
à medida que se aumentou a dose do extrato de pimenta-do-reino. Esse fato se deve provavelmente aos efeitos alelopáticos do extrato, que foram tornando-se maiores com o passar do tempo. Segundo RICE (1984) e VERONKA (2011), esse efeito alelopático refere-se a qualquer efeito direto ou indireto, benéfico ou prejudicial, de uma planta sobre outra planta, mediante produção de compostos químicos (aleloquímicos) que são liberados no ambiente.

Ainda são pouco conhecidos os mecanismos de ação dos agentes alelopáticos, dada à dificuldade de se separar os efeitos secundários das causas primárias, segundo BERTOLINI et al. (2005). No entanto, estudos como o do autor RICE (1984) já demonstram a existência de efeitos sobre a divisão, alongamento e ultraestrutura celulares, crescimento induzido por hormônios, permeabilidade de membranas, absorção mineral, abertura estomática, fotossíntese, respiração, síntese proteica, metabolismo de lipídios e ácidos orgânicos, atividade de várias enzimas e nas relações hídricas do vegetal. As alterações que as sementes podem sofrer a ponto de diminuir o seu potencial germinativo, deve-se segundo FERREIRA \& AQUILA (2000), aos efeitos sobre a permeabilidade das membranas, a respiração, a conformação de enzimas e de receptores, entre outros, ou, ainda, pela combinação desses fatores.

TABELA 1. Interação do tempo com taxa de germinação das sementes tratadas com extrato de pimenta-do-reino em diferentes quantidades em (\%).

\begin{tabular}{cccccc}
\hline & \multicolumn{5}{c}{ Tempos (Dias) } \\
\cline { 2 - 6 } Doses $(\mathrm{mL})$ & 30 & 60 & 90 & 120 & 150 \\
\hline 0 & $100,00 \mathrm{aA}$ & $79,00 \mathrm{bB}$ & $62,00 \mathrm{aC}$ & $13,50 \mathrm{aD}$ & $9,50 \mathrm{aE}$ \\
10 & $100,00 \mathrm{aA}$ & $82,50 \mathrm{aB}$ & $35,00 \mathrm{bC}$ & $10,00 \mathrm{bD}$ & $7,00 \mathrm{aD}$ \\
20 & $100,00 \mathrm{aA}$ & $71,50 \mathrm{cB}$ & $24,50 \mathrm{cC}$ & $0,00 \mathrm{cD}$ & $0,00 \mathrm{bD}$ \\
\hline DMS (Linha) & \multicolumn{5}{c}{3,75} \\
\hline DMS (Coluna) & \multicolumn{7}{c}{3,19} & \\
\hline
\end{tabular}

Classificação com letras minúsculas para coluna; Classificação com letras maiúsculas para linha; Caracterização da germinação $=100,00 \%$.

Para uma resposta matemática, os dados quantitativos foram submetidos a uma análise de regressão (Figura 1). O estudo das equações lineares revelou efeito significativo e $\mathrm{R}^{2}$ acima de $91 \%$ para equação de primeiro grau, indicando que esta pode ser utilizada para representar o comportamento da germinação de sementes de soja tratadas com extrato de pimenta-do-reino. Em análise na Figura 1, observase que para as sementes tratadas com o extrato nas diferentes doses ( $\mathrm{mL}$ ) houve perda de germinação durante o armazenamento e que essa perda se acentua à medida que avança o tempo de armazenagem. As sementes de soja que apresentaram uma germinação inicial de $100 \%$ chegaram no final do armazenamento (150 dias) com uma germinação de $9,50 \%$ na dose de $0 \mathrm{~mL}$ (testemunha), 7,0\% na dose de $10 \mathrm{~mL}$ e $0,0 \%$ de germinação na dose de $20 \mathrm{~mL}$.

LACERDA et al. (2003) avaliando as características fisiológicas, bioquímicas e sanitárias das sementes de soja armazenadas por seis meses e tratadas com um fungicida, observaram que as sementes não obtiveram índices de emergência suficientes para comercialização. A porcentagem de germinação de sementes armazenadas e tratadas com fungicidas foram inferiores aos das sementes não tratadas, podendo o fungicida utilizado, ter influenciado negativamente em fases do 
processo de germinação, mesmo sendo este recomendado no tratamento de sementes de soja.

É importante ressaltar que a semente de soja só poderia ser comercializada até os 60 dias de armazenagem, período em que apresentou germinação acima de $70 \%$. Os resultados expressos nos demais períodos, não atende a legislação vigente para a semente que exige germinação acima de 70\% CESM (1989).

Segundo ALMEIDA et al. (2004) as sementes ao atingirem sua máxima qualidade fisiológica, entra num processo contínuo e irreversível de deterioração não pode ser evitado. Entretanto, pode decrescer de maneira lenta, quando armazenadas adequadamente.

QUEIROGA et al. (2012) que ao estudarem a viabilidade de sementes de feijão carioca tratadas com óleos fixos de mamona, soja e oiticica durante um armazenamento de 5 meses, observaram diminuição no percentual de germinação dessa semente com o avanço da armazenagem.

$0 \mathrm{~mL}$

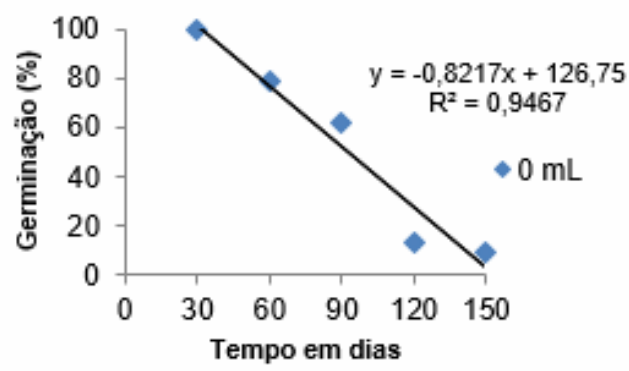

(A)

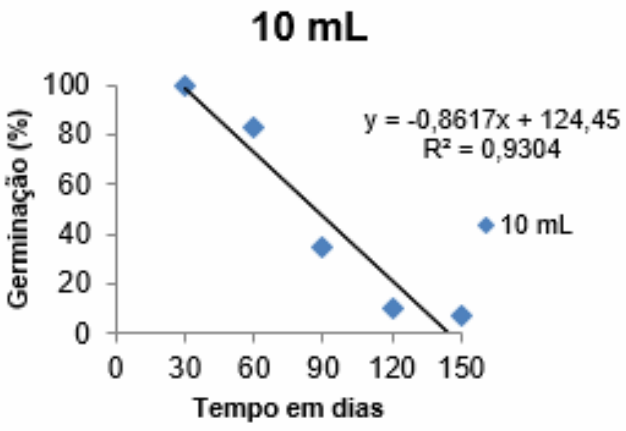

(B)

$20 \mathrm{~mL}$

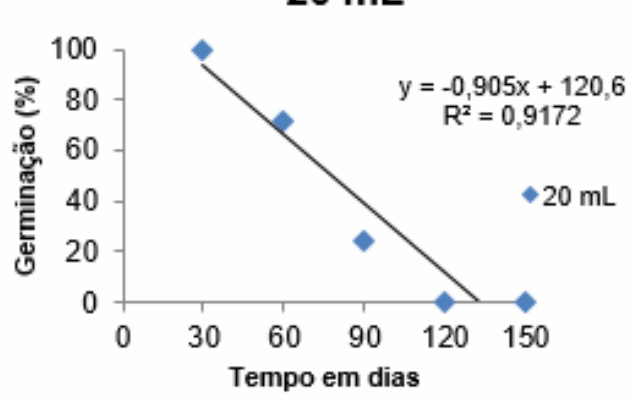

(C)

FIGURA 1. Germinação (\%) das sementes de soja ao longo de 150 dias de armazenamento, tratadas com extrato de Piper nigrum nas doses (A) $0 \mathrm{~mL}$, (B) 10 $\mathrm{mL}$ e (C) $20 \mathrm{~mL}$.

Os fungos que se desenvolveram sobre as sementes de soja armazenadas, foram Aspergillus flavus e Penicillium sp., com maior incidência do Penicillium frente ao Aspergillus flavus nas sementes armazenadas por 150 dias, a exceção se deu no tempo de 120 dias para dose de $20 \mathrm{~mL}$. CHRISTENSEN (1974) relata que os fungos de armazenamento compreendem várias espécies grupais do gênero Aspergillus e Penicillium. A específica composição fúngica no armazenamento é altamente 
dependente do conteúdo de água da semente, que mesmo sofrendo pequenas variações, determina modificações substanciais.

TABELA 2. Incidência do fungo Penicillium sp. e Aspergillus flavus nas sementes tratadas com extrato de pimenta-do-reino em diferentes doses em (\%).

\section{Penicillium sp.}

\begin{tabular}{cccccc}
\hline \multirow{2}{*}{ Doses $(\mathrm{mL})$} & \multicolumn{5}{c}{ Tempos (Dias) } \\
\cline { 2 - 6 } & 30 & 60 & 90 & 120 & 150 \\
\hline 0 & $0,00 \mathrm{aA}$ & $0,00 \mathrm{aA}$ & $0,00 \mathrm{aA}$ & $0,00 \mathrm{cA}$ & $0,00 \mathrm{cA}$ \\
10 & $0,00 \mathrm{aC}$ & $0,00 \mathrm{aC}$ & $0,00 \mathrm{bC}$ & $35,00 \mathrm{bB}$ & $42,50 \mathrm{bA}$ \\
20 & $0,00 \mathrm{aC}$ & $0,00 \mathrm{aC}$ & $0,00 \mathrm{aC}$ & $65,00 \mathrm{aB}$ & $72,50 \mathrm{aA}$ \\
\hline DMS (Linha) & \multicolumn{5}{c}{5,60} \\
\hline DMS (Coluna) & \multicolumn{5}{c}{ Aspergillus flavus } \\
\hline \multicolumn{5}{c}{ Tempos (Dias) } \\
\hline Doses (mL) & 30 & 60 & 90 \\
\cline { 2 - 6 } & $0,00 \mathrm{aA}$ & $10,00 \mathrm{aA}$ & $0,00 \mathrm{aA}$ & $0,00 \mathrm{aD}$ & $0,00 \mathrm{aA}$ \\
10 & $0,00 \mathrm{aB}$ & $0,00 \mathrm{bB}$ & $0,00 \mathrm{aB}$ & $7,50 \mathrm{bB}$ & $42,40 \mathrm{bA}$ \\
20 & $0,00 \mathrm{aC}$ & $0,00 \mathrm{bC}$ & $0,00 \mathrm{aC}$ & $85,00 \mathrm{aA}$ & $52,50 \mathrm{aB}$ \\
\hline DMS (Linha) & \multicolumn{5}{c}{10,91} \\
\hline DMS (Coluna) & \multicolumn{5}{c}{9,30} \\
\hline Con
\end{tabular}

Classificação com letras minúsculas para coluna; Classificação com letras maiúsculas para linha; Caracterização Penicillium e A. flavus $=0,00 \%$

É importante observar que a maior incidência dos fungos Penicillium e $A$. flavus se deu a partir dos 120 dias de armazenamento para as sementes que receberam as doses de 10 e $20 \mathrm{~mL}$ do extrato de pimenta do reino, indicando que o extrato pode ter estimulado o crescimento dos fungos. Esses resultados estão de acordo com os de VENTUROSO (2005) que relatam um estímulo ao crescimento conferido pelo extrato de cavalinha.

Estes resultados são contrários aos de GARCIA et al. (2012) que estudando o efeito de óleos e extratos vegetais sobre o crescimento micelial de $S$. sclerotiorum, observaram que dentre os extratos vegetais aquosos avaliados, o fruto de pimenta longa foi o mais promissor na inibição do crescimento micelial.

De acordo com BARRETO et al. (2003), os princípios ativos são oriundos do metabolismo secundário das plantas. Esses compostos são produzidos como mecanismo de defesa da planta contra fatores externos e, sobremaneira, contra patógenos, nos quais se destacam os fungos. Essas propriedades são dependentes de uma série de fatores inerentes às plantas, como órgão utilizado, idade e estágio vegetativo, fatores do ambiente como pH do solo, estação do ano e diferentes tipos de estresse que as plantas sofrem.

Ainda em relação aos dados contidos na Tabela 2, é importante notar que a quantidade de fungos presente na semente, para as doses de 10 e $20 \mathrm{~mL}$ foram maiores que a dose de $0 \mathrm{~mL}$ (testemunha). Esse fato deve-se provavelmente a quantidade de água ser maior nas sementes armazenadas com o extrato (Tabela 3) em relação às sementes não tratadas. A ação desses microrganismos pode ser acelerada desde que haja condições de umidade e temperatura o que ocorreu no experimento, quando a partir dos 90 dias ocorreram alterações na umidade das sementes. 
TABELA 3. Valores médios da umidade (\%) das sementes de soja tratadas com extrato de pimenta-do-reino em diferentes doses e armazenadas por 150 dias em ambiente não controlado

\begin{tabular}{ccc}
\hline & Doses & \\
\hline 0 (testemunha) & $10 \mathrm{~mL}$ & $20 \mathrm{~mL}$ \\
\hline $8,75 \mathrm{~b}$ & $9,04 \mathrm{ab}$ & $9,57 \mathrm{a}$ \\
\hline DMS & 0,60 & \\
\hline
\end{tabular}

Caracterização da umidade antes do armazenamento: sementes não tratadas 8,90\%; sementes tratadas com $10 \mathrm{~mL} 9,19 \%$ e sementes tratadas com $20 \mathrm{~mL} 9,24 \%$

\section{CONCLUSÃO}

O extrato de pimenta-do-reino é eficiente na manutenção da viabilidade das sementes até 60 dias de armazenamento.

$O$ extrato de pimenta-do-reino apresenta eficiência em controlar o fungo $A$. flavus aos 60 dias em relação às sementes não tratadas.

\section{REFERÊNCIAS BIBLIOGRÁFICAS}

ALMEIDA, F. A. C.; FIGUEIRÊDO NETO, A.; COSTA, R. F.; GOUVEIA, J. P. G.; OLIVEIRA, M. E. C. Danos mecânicos em sementes de feijão Vigna, causados pelas operações na unidade de beneficiamento. Revista Brasileira de Engenharia Agrícola e Ambiental, v. 8, n. 2-3, p. 254-259, 2004.

BARRETO, A. F.; ARAÚJO, E.; BELTRÃO, N. E. M.; DIONÍZIO, G. Análise sanitária de sementes de algodão (Gossypium hirsutum L. r. latifolium Hutch) da cultivar BRS 201 tratadas com extrato de agave. In: Congresso Brasileiro de Algodão, 4, 2003, Goiânia, Resumos. 2003. CD-ROM.

BERTOLINI, M. F. FORTES, A. M. T. Efeitos alelopáticos sobre a germinação de sementes de soja (Glycine Max L.Merrill). Semina: Ciências Agrárias, v. 26, n. 1, p. 5-10, 2005.

BRASIL, Ministério da Agricultura e Reforma Agrária. Regras para Análise de sementes. Brasília: SNDA/DNDV/CLAV, 2009. 365p.

CARVALHO, N. M.; NAKAGAWA, J. Sementes: ciência, tecnologia e produção. 5.ed. Jaboticabal: FUNEP, 2012. 590p.

CESM - Comissão Estadual de Sementes e Mudas. Normas técnicas para produção de sementes e mudas fiscalizadas. João Pessoa: Delegacia Federal de Agricultura da Paraíba - Seapro (DFA/PB), 1989.

CHRISTENSEN, C. M. Micoflora and seed deterioration. In: Roberts, E. H. Viability of seeds. London, Chapman and Hall, 1974. p. 59-93.

CONAB - Companhia Nacional de Abastecimento. Acompanhamento da safra brasileira de grãos. v.2 - SAFRA 2014/15 N.5 - Nono Levantamento junho/2015. Disponível em:<http://www.conab.gov.br/OlalaCMS/uploads/arquivos/15_06_11_09_00_38_bol etim_graos_junho_2015.pdf>. Acesso em: 26 jun. 2015. 
DEUNER, C.; ALMEIDA, A. S.; BORGES, C. T.; JAUER, A.; MENEGHELLO, G. E. Efeito de produtos químicos aplicados via tratamento de semente de soja. Enciclopédia Biosfera, v.11, n.21, p.863-868, 2015.

FERREIRA, A.G. \& AQÜILA, M.E.A. Alelopatia: Uma área emergente da ecofisiologia. Revista Brasileira Fisiologia Vegetal, v.12, p.175-204, 2000.

GARCIA, R. A.; JULIATTI, F. C.; BARBOSA, K. A. G.; CASSEMIRO, T.A. ATIVIDADE ANTIFÚNGICA DE ÓLEO E EXTRATOS VEGETAIS SOBRE Sclerotinia sclerotiorum. Bioscience Journal, v. 28, n. 1, p. 48-57, Jan./Feb. 2012.

LACERDA, A. L. DE S.; LAZARINI, E.; SÁ, M. E.DE; VALÉRIO FILHO, V.W. Armazenamento de sementes de soja dessecadas e avaliação da qualidade fisiológica, bioquímica e sanitária. Revista Brasileira de Sementes, v. 25, n. 2, p.97-105, 2003.

MIGLIORINI, P.; KULCZYNKI, S. M.; SILVA, T. A.; BELLÉ, C.; KOCH, F. Efeito do tratamento químico e biológico na qualidade fisiológica e sanitária de sementes de canola. Enciclopédia Biosfera, v.8, n.15, p.789-801, 2012.

NEERGAARD, P. Seed pathology. London: Mac Millan, v.2, 839p, 1979.

QUEIROGA, M. de F. C. de; GOMES, J. P.; ALMEIDA, F. de A. C.; PESSOA, E.B.; ALVES, N. M. C. Aplicação de óleo no controle de Zabrotes subfasciatus e na germinação de Phaseolus vulgaris. Revista Brasileira de Engenharia Agrícola e Ambiental, v.16, n.7, p.777-783, 2012.

RICE, E. L. Allelopathy. 2 ed New York: Academic Press, 1984. 422p.

SCHNEIDER, C. F.; GUSATTO, F. C.; MALAVASI, M. DE M.; STANGARLIN, J. R.; MALAVASI, U. C.; Termoterapia na qualidade fisiológica e sanitária de sementes armazenadas de pinhão-manso. Semina: Ciências Agrárias, v. 36, n. 1, p. 47-56, 2015.

SMANIOTTO, T. A. S.; RESENDE, O.; MARÇAL, K. A. F.; OLIVEIRA, D. E. C.; SIMON, G. A. Qualidade fisiológica das sementes de soja armazenadas em diferentes condições. Revista Brasileira de Engenharia Agrícola e Ambiental, v.18, n. 4, p. 446-456, 2014.

SILVA, M. S.; NAVES, M. M. V.; OLIVEIRA, R. B. DE; LEITE, O. DE S. M. Composição química e valor proteico do resíduo de soja em relação ao grão de soja. Revista Ciência e Tecnologia de Alimentos, v.26, n.3, 2006.

VENTUROSO, L. R.; BACCHI, L. M. A.; GAVASSONI, W. L.; CONUS, L. A.; PONTIM, B. C. A.; BERGAMIN, A. C. Atividade antifúngica de extratos vegetais sobre o desenvolvimento de fitopatógenos. Summa Phytopathologica, v. 37, p. 1823, 2011. 
VERONKA, D.A. Alelopatia do extrato bruto de brachiaria decumbens na germinação de sementes e no vigor de sementes e de plântulas de Brachiaria brizantha. Dissertação (mestrado em Meio Ambiente e Desenvolvimento Regional) Universidade Anhanguera - Uniderp, 39f. 2011. 